a process called translation - are positively correlated with temperature. Experimental studies in the laboratory show that the translation apparatus of two diatom species worked most efficiently when grown at temperatures close to average equatorial surface waters, and were less efficient at Arctic temperatures as the cold slowed down the molecular machinery. However, Toseland et al. observed that actual cellular productivity in the Arctic and Antarctic was not as repressed as it should be, despite the colder water. They attribute this to a considerable increase in abundance of the cellular translation machinery that helps to build protein, so called ribosomes, which are bound in P-rich RNA (Fig. 1). Hence, to overcome the low water temperatures (average of $2{ }^{\circ} \mathrm{C}$ ) and concomitant reduction in efficiency, these cells just make more protein factories to maintain their productivity. As this requires more $\mathrm{P}$, the $\mathrm{N}: \mathrm{P}$ ratio in their cells is reduced.

This information led to the development of a physiological model of the phytoplankton cell that described how much available $\mathrm{P}$ and $\mathrm{N}$ the cell would use for creating protein, versus how much it would put into creating RNA. The problem is that RNA uses more P, which is often a limiting nutrient in the world's oceans ${ }^{3}$; therefore if the cell diverts its resources to create more RNA-laden ribosomes to overcome their reduced efficiency, it needs more $\mathrm{P}$ than cells found in warmer water at the equator. The authors placed their model cell in a computer-generated model ocean that replicates the changing temperature, nutrient availability and amount of light that real phytoplankton cells would experience across the global ocean. The model validated the hypothesis that under low temperatures the cells invested more in their cellular machinery to overcome the inefficiency of their factories; whereas under higher temperatures the cells invested in photosynthesis and hence biomass.

In further work they artificially raised the average sea surface temperature by $5{ }^{\circ} \mathrm{C}$, and observed what happened to the phytoplankton cell. As the polar sea warmed up, the phytoplankton cell reduced the production of P-rich ribosomal RNA, changing the cellular N:P ratio, which by definition fundamentally alters this ratio in organic matter. Why does this matter? If the $\mathrm{N}: \mathrm{P}$ ratio increases then the cell has an increased $\mathrm{N}$ requirement, which will cause $\mathrm{N}$ to become a limiting resource. Nitrogen limitation could reduce photosynthetic productivity causing an increase in carbon flux from the surface ocean to the atmosphere, thereby resulting in a significant reduction in carbon sequestration by the ocean. Potentially this could result in a catastrophic positive feedback loop, as more atmospheric carbon equals more warming ${ }^{9}$.

Although this model represents one of the most sophisticated methods for capturing and predicting the result of rising temperature on global oceanic primary productivity, it still has limitations. For example, it doesn't take into consideration the changes in atmospheric carbon levels, which could bolster photosynthetic efficiency and inflate predictions. The model also doesn't account for cyanobacteria, the other major phytoplankton group in the ocean, nor the interactions with other non-photosynthetic bacteria. Future work should focus on the integration of these efforts to create a comprehensive model that will enable us to predict the real outcome of climate change and global warming in this essential system.

Jack A. Gilbert is at Argonne National Laboratory, 9700 South Cass Avenue, Lemont, Illinois 60439, USA and University of Chicago, 9700 South Cass Avenue, Lemont, Illinois 60439, USA.

e-mail: gilbertjack@uchicago.edu

References

1. Toseland, A. et al. Nature Clim. Change 3, 979-984 (2013).

2. Jorgensen, B. B. \& Boetius, A. Nature Rev. Microbiol. 5, 770-781 (2007)

3. Falkowski, P. G., Barber, R. T. \& Smetacek, V. Science 281, 200-206 (1998)

4. Field, C. B., Behrenfeld, M. J., Randerson, J. T. \& Falkowski, P. G. Science 281, 237-240 (1998)

5. Bopp, L., Aumont, O., Cadule, P., Alvain, S. \& Gehlen, M. Geophys. Res. Lett. 32, L19606 (2005).

6. Shuter, B. J. Theor. Biol. 78, 519-552 (1979).

7. Follows, M. J., Dutkiewicz, S., Grant, S. \& Chisholm, S. W. Science 315, 1843-1846 (2007).

8. Weber, T. S. \& Deutsch, C. Nature 467, 550-554 (2012)

9. Martiny, A. C. et al. Nature Geosci. 6, 279-283 (2013)

\title{
Big data insights into pest spread
}

Pests and diseases reduce agricultural yields and are an important wildcard in the evaluation of future climate impacts. A unique global record of pests and diseases provides evidence for poleward expansions of their distributions.

\section{Karen A. Garrett}

Fis: ood security depends on our ability to effectively manage crop pests (arthropods and pathogens). Because of the important effects of weather variables such as temperature and precipitation on crop pests, scientists have for some time hypothesized that where climate change results in a more (less) favourable environment for pest establishment, losses to unmanaged pests are likely to increase (decrease) ${ }^{1}$. But evidence that ranges have shifted under climate change is often anecdotal, and the availability of long-term data sets of pest occurrence is limited ${ }^{2,3}$. In this issue of Nature Climate Change, Bebber and colleagues ${ }^{4}$ present an analysis of decades of reported pest distributions, concluding that pests have moved towards the poles over the past fifty years, in line with expectation under climate change.

One of the interesting aspects of this analysis is its reliance on 'big data. The data set that Bebber and colleagues ${ }^{4}$ analysed, although not challenging in terms of sheer storage and computational requirements, has been assembled over some time as many, many individuals reported where and when they found particular pests. In their popular book, Mayer-Schönberger and Cukier ${ }^{5}$ discuss three aspects of big data that present challenges for scientists. The first is a shift towards using large amounts of data from different sources, often collected for different purposes. The second is an acceptance of 'messiness', where having large amounts of data may make up for introducing increased sources of variability, and potentially even for introducing bias (more on that later). The 


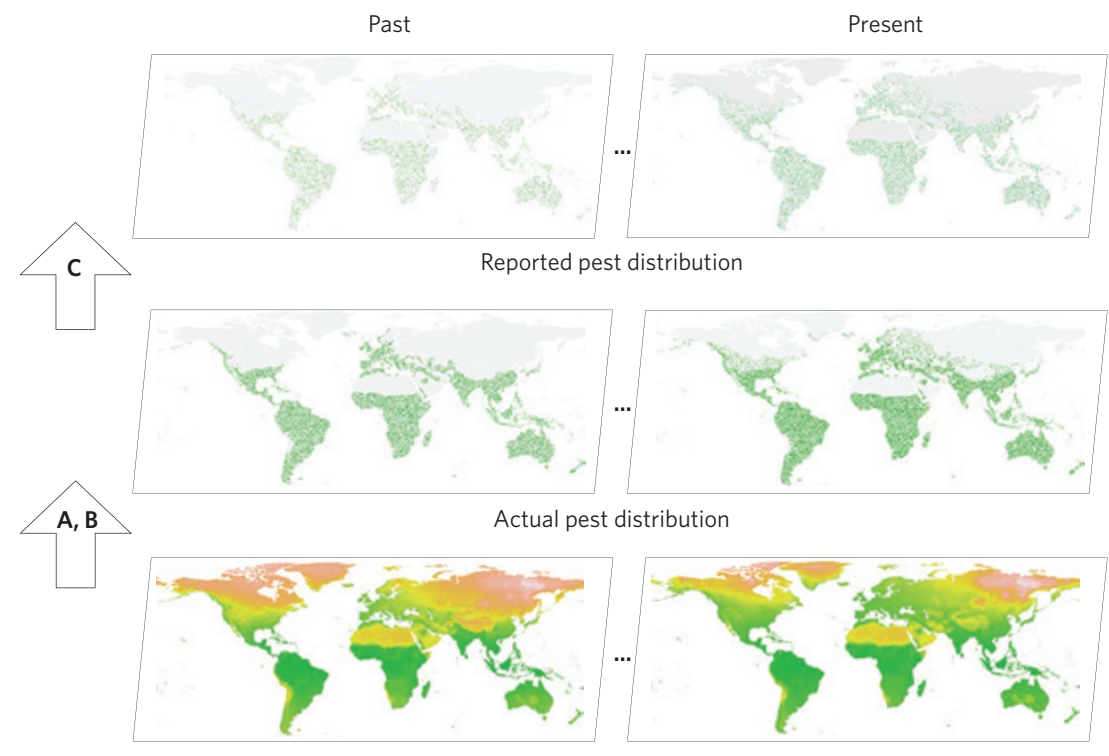

Climate favourability for a pest
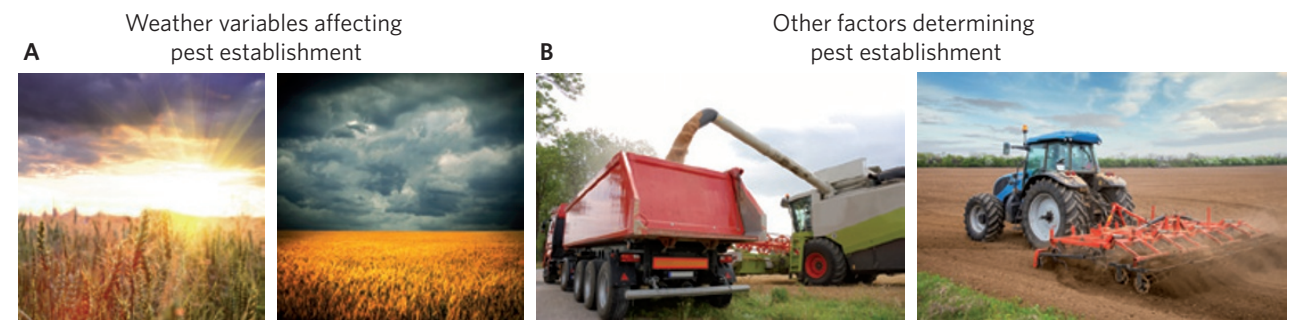

Challenges for interpreting pest observations

Problem of lack of zeroes:

People rarely carefully evaluate and report the absence of a pest

Many factors determine the distribution of pests, and the reported distribution of pests There is the potential for unknown forms of bias in observations

Figure 1 | Bebber and colleagues ${ }^{4}$ address the challenge of evaluating how rapidly crop pests (arthropods and pathogens) have spread to the north or south over recent decades, based on observations accumulated from a wide range of observers. The maps show an example for a hypothetical pest. A, Weather variables such as temperature, precipitation and relative humidity are important in determining how likely a pest is to become established in a new area, influencing risk factors such as the probability of overwintering (oversummering) and the number of generations per year. B, Other factors that determine how likely a pest is to become established in a new area include: human transportation networks; agricultural management, including planting dates and use of tillage; the distribution of susceptible hosts, where some forms of resistance are temperature-sensitive; interactions with other hosts, arthropods and microbes. C, Other factors that determine how likely an established pest is to be observed include: traits of pest or disease (ease of identification or diagnosis, perceived economic importance, shifts in taxonomic resolution); traits of host (economic importance, hectarage); traits of location (infrastructure, training of personnel, sampling effort). Images from: A,B, Istock/Thinkstock; C, (c) E. De Wolf.

third is a willingness to accept correlations as the outcome of analyses, rather than necessarily understanding causation.

Big data issues are not new in the context of mapping the geographic distribution of species $^{6}$. Pest risk mapping generally has as a first step either (1) recording where a pest has been observed and the associated environmental conditions, or (2) obtaining estimates of the effects of environmental variables on a pest in controlled experimental conditions such as growth chambers ${ }^{7}$. Both approaches may err by estimating the environment-risk relationship based on specific factors (such as sampling approaches or pest subpopulations) that are not relevant in other areas where risk will be evaluated. A particular problem with the records of presence, as in the data used by Bebber and colleagues, is that there tends to be little information about the uncertainty associated with these records, in contrast to some more standard approaches to meta-analysis.

Bebber and colleagues ${ }^{4}$ take geographic mapping a step further by evaluating how the records of pest distributions have changed over time. An important challenge in interpreting the records in this way is to convincingly demonstrate that sources of bias have been adequately accounted for, or that the analysis is conservative with regard to likely sources of bias. Bebber and colleagues use the assumption that the scientific infrastructure in tropical nations has lagged that in temperate areas to postulate that more limited sampling effort in the tropics may result in a bias towards reporting pests in temperate countries earlier. Consequently, when they find that reporting has increased in temperate areas in recent years, this is conservatively interpreted as evidence that pests are moving further into temperate regions. This is a key argument, but one that is difficult to evaluate because good records of sampling effort corresponding to entries in the CABI database ${ }^{8}$ would be very difficult to assemble. It can be argued, however, that the CABI data set is such a unique entity, as the largest global repository for data related to global pest distributions, that it merits analysis even if we know there is the possibility of little-understood biases.

An understanding of current and future changes in the geographic ranges of agricultural pests is important from several standpoints. Good estimates of current changes allow us to test our understanding 
of the most important factors influencing pest risk, and improve our ability to provide realistic scenarios for the future. Scenarios of future pest and disease distributions under climate change support prioritization in agricultural research programmes. For example, if it is likely that a particular disease will become more important in a region, crop-breeding programmes can respond by incorporating better resistance to that disease in locally adapted varieties.

The prevalence of crop pests is a function of many factors, so identifying particular drivers of change is challenging ${ }^{9}$ (Fig. 1). If we wish to ask whether crop pests have altered distributions because of climate change, this is complicated by the many other factors that have changed simultaneously, even when long-term data are available ${ }^{10}$. It is reasonable to expect that higher temperatures will often reduce limitations on pest overwintering and increase the number of pest generations per year (Fig. 1A). However, other factors simultaneously influence pest risk at any location, including host genotypic and phenotypic resistance, where phenotypic resistance may respond to weather variables such as temperature (Fig. 1B). When multiple observers and multiple levels of sampling effort are involved, this adds another layer of uncertainty when comparing the actual distribution of pests and reported distributions (Fig. 1C).

Bebber and colleagues ${ }^{4}$ provide a stimulating analysis of changes in pest distributions, along with a new set of hypotheses to engage scientists working with pests. Future 'big data' analyses may address the geographic distribution of pest genomes and microbial metagenomes associated with plants and soil, including analysis of the geographic spread of genes important in crop damage. Cell phone availability may facilitate analysis of global digital images of crop damage. Better data archiving systems and more data sharing are needed to support future synthetic analyses. For addressing large-extent questions, we also need advances in methods to evaluate more directly the factors that lead, not only to pest risk, but also to reporting of observations, to support understanding of what variables may be good proxies for sampling effort.

Karen A. Garrett is in the Department of Plant Pathology, Kansas State University, Manhattan, Kansas 66506, USA.

e-mail:kgarrett@ksu.edu

References

1. Coakley, S. M., Scherm, H. \& Chakraborty, S. Ann. Rev. Phytopathol. 37, 399-426 (1999)

2. Jeger, M. J. \& Pautasso, M. New Phytol. 177, 8-11 (2008).

3. Shaw, M. W. \& Osborne, T. M. Plant Pathol. 60, 31-43 (2011)

4. Bebber, D. P., Ramotowski, M. A. T. \& Gurr, S. J. Nature Clim. Change 3, 985-988 (2013).

5. Mayer-Schönberger, V. \& Cukier, K. Big Data: A Revolution That Will Transform How We Live, Work, and Think (Houghton Mifflin Harcourt, 2013).

6. Sutherst, R. W. J. Biogeogr. 30, 805-816 (2003).

7. Venette, R. C. et al. BioScience 60, 349-362 (2010).

8. Pasiecznik, N. M. et al. EPPO Bull. 35, 1-7 (2005).

9. Garrett, K. A. et al. Plant Pathol. 60, 15-30 (2011).

10. Hannukkala, A. O., Kaukoranta, T., Lehtinen, A. \& Rahkonen, A. Plant Pathol. 56, 167-176 (2007).

\section{Bottom up in the tropics}

A study reveals that recent warming in the Indian Ocean and in the Pacific 'warm pool' has caused a cooling near the top of the tropical troposphere above, leading to less water vapour entering the stratosphere.

\section{Qiang Fu}

W

ater vapour in the stratosphere is a greenhouse gas. It is constrained from entering the stratosphere in the tropics by the thermal boundary between the stratosphere and troposphere $^{1}$ - the tropical tropopause, the coldest point in the lower atmosphere. Cold-point temperatures at the tropical tropopause (Fig. 1a) have important implications for both stratospheric chemistry $^{2}$ and global climate change ${ }^{3}$. The importance of the spatial distribution of temperature (Fig. 1b) is well recognized, as the temperature minimum is relevant to cloud formation and subsequent dehydration through atmospheric circulation $^{4}$. In the boreal winter, for example, the lowest cold-point temperatures over the warm pool in the tropical western Pacific govern the amount of water vapour that enters the stratosphere ${ }^{5}$. It is thus critically important to understand how the zonal (longitudinal) structure of the tropical cold-point temperature would respond to global warming. Now, reporting in the
Journal of Geophysical Research, Garfinkel and co-workers ${ }^{6}$ find that the warming in the tropical upper troposphere over the past 30 years has been strongest over the Indo-Pacific warm pool, where cooling near the tropopause has been strongest. They suggest that warming in the Indian Ocean and the Pacific warm pool has led to zonal asymmetry in atmospheric temperature trends, and that such trends may continue in the future.

Temperatures near the tropical tropopause are determined by a complex combination of stratospheric (top-down) and tropospheric (bottom-up) processes ${ }^{7}$. The zonal structures at $100 \mathrm{hPa}$ (Fig.1b) closely resemble the mean pattern of the equatorial planetary waves - large-scale perturbations of the atmospheric dynamical structure. These are driven by massive convection over the Indo-Pacific warm $\mathrm{pool}^{8}$, with the lowest temperatures and largest cirrus cloud fractions over the western Pacific and Maritime Continent (which includes the islands of Indonesia,
New Guinea and Malaysia, and the surrounding shallow seas) ${ }^{9}$. The signature of the equatorial planetary waves is also evident in the temperature variability over intraseasonal to interannual timescales ${ }^{9}$. The responses of temperature structures at 100 and $250 \mathrm{hPa}$ are reversed in sign because the maximum amplitude of equatorial planetary waves with opposite phases occurs at these two levels. The temperatures and cloud fraction near the tropical tropopause are also strongly modulated by extra-tropical stratospheric waves. These drive the Brewer-Dobson circulation (BDC) - a large-scale latitudinal circulation in the stratosphere with air rising across the tropical tropopause, moving polewards and sinking towards the extra-tropical troposphere - which is particularly evident in their seasonal cycles $^{9-11}$ (Fig.1c). In contrast to the equatorial planetary waves, the extra-tropical stratospheric waves are associated with zonally symmetric temperature anomalies in the lower stratosphere. 